Research Article

\title{
Chaihu plus Longgu Muli Decoction Alleviated Brain Injury in Pentylenetetrazole-Kindled Epileptic Mice by Regulating Cyclooxygenase-2/Prostaglandin E2/Multidrug Transporter Pathway
}

\author{
Ping Shan $\left(\mathbb{D}^{1},{ }^{1}\right.$ Jilong Zhang, ${ }^{2}$ Yulan Gou, ${ }^{1}$ Lijun Luo, ${ }^{1}$ and Suiqiang Zhu $\mathbb{D}^{3}$ \\ ${ }^{1}$ Department of Neurology, Wuhan First Hospital, Wuhan 430022, China \\ ${ }^{2}$ Department of Emergency, Wuhan First Hospital, Wuhan 430022, China \\ ${ }^{3}$ Department of Neurology, Tongji Hospital, Tongji Medical College of Huazhong University of Science and Technology, \\ Wuhan 430030, China \\ Correspondence should be addressed to Ping Shan; spring12w@sina.com and Suiqiang Zhu; zhusuiqiang@163.com
}

Received 21 October 2020; Revised 29 January 2021; Accepted 2 March 2021; Published 30 March 2021

Academic Editor: Steven De Vleeschouwer

Copyright (c) 2021 Ping Shan et al. This is an open access article distributed under the Creative Commons Attribution License, which permits unrestricted use, distribution, and reproduction in any medium, provided the original work is properly cited.

Objective. To evaluate the effect of CLMD administration on epileptic seizures and brain injury in pentylenetetrazole- (PZT-) kindled mice. Methods. The effect of pretreatment with CLMD (5, 10, and $20 \mathrm{ml} / \mathrm{kg}(\mathrm{mg} / \mathrm{kg})$ by gavage) for seven days on PTZinduced kindling, duration and grade of kindling-induced seizures, and pathological injury in the cortex and hippocampus was evaluated. Male BALB/c mice with adenosine A1 receptor knockout were subjected to intraperitoneal injection of PTZ $(35 \mathrm{mg} / \mathrm{kg})$ once every day until kindling was successfully induced. Quantitative reverse transcription polymerase chain reaction, immunofluorescence, and western blot were performed to assess the mRNA and protein levels of p-glycoprotein (PGP), multidrug resistance-associated protein 1 (MRP1), cyclooxygenase-2 (COX-2), prostaglandin E2 (PGE2), and adenylate kinase $(\mathrm{ADK})$ in the cortex and hippocampus. Results. PTZ successfully induced kindling in mice after 21 days, wherein CLMD showed an obvious dose-dependent antiepileptic effect. High-dose CLMD significantly increased the latency of epileptic seizures, decreased the sustained time of epileptic seizures and the seizure grade, and ameliorated the histopathological changes in the cortex and hippocampus. Furthermore, PTZ kindling induced significantly higher levels of PGP, MRP1, COX-2, PGE2, and ADK, but this effect was inhibited by pretreatment with CLMD in a dose-dependent manner. Conclusion. Pretreatment with CLMD attenuates PTZ-kindled convulsions and brain injury in mice. The mechanism may be related to the cyclooxygenase2/prostaglandin E2/multidrug transporter pathway.

\section{Introduction}

Epilepsy is one of the most common and disabling neurological diseases and is characterized by recurrent seizure activity [1]. According to epidemiological studies, approximately 65 million people worldwide suffer from epilepsy, and the incidence in China accounts for approximately one-tenth of the total epileptic population [2]. In spite of the clinical application of around 20 antiepileptic drugs (AEDs), $20-30 \%$ of patients still experience refractory epilepsy because of the unsatisfactory efficacy of these drugs [3]. This results in increased mortality and disability rates, declining quality of life, and increasing social and family economic burden. Therefore, it is imperative to explore new prevention and treatment strategies for refractory epilepsy.

In recent years, the determination of effective concentrations of AEDs at traumatic brain lesions has become a key in the prevention of refractory epilepsy [4]. Multidrug transporters (MDTs), such as p-glycoprotein (PGP) and multidrug resistance-associated protein 1 (MRP1) in the central nervous system, are mainly expressed on the lumen surface of cerebral capillary endothelial cells and are associated with 
the role of adenosine triphosphate- (ATP-) dependent efflux pumps [5]. They can be combined with a variety of AEDs in the cell and be pumped into blood circulation, limiting the accumulation of lipid-soluble AEDs in the brain and thereby maintaining the stability of the brain environment [6]. Common first-line AEDs, such as phenytoin, carbamazepine, and phenobarbital, have been identified as transport substrates of PGP and MRPs [7]. However, the overexpression of MDTs can limit the uptake and absorption of AEDs in brain tissue and reduce the drug concentration in the brain, thus obstructing the drug from reaching the target and reducing drug efficacy [8]. Therefore, inhibiting the high expression of MRPs to optimize the concentration of AEDs in diseased brain tissues has become a highlight in refractory epilepsy research.

Numerous studies have shown that epileptic seizures are accompanied by inflammatory response and that persistent seizures produce chronic inflammation $[9,10]$. Recent studies have reported that the inflammatory response induced by epilepsy is closely related to the activation of cyclooxygenase-2 (COX-2) and the increase in prostaglandins (PGs) $[11,12]$. Indeed, arachidonic acid is released from membrane phospholipids during seizures and is oxidized by COX and lipoxygenase, generating proinflammatory products [13]. COX-2 is a speed-limiting enzyme synthesized by PGs that not only generates oxidative stress after activation but also converts arachidonic acid into PGs and other harmful substances and promotes repeated seizures [14]. Upregulated COX-2 expression was reported in epileptic seizures, and the activation of COX-2 in the brain of epileptic animals promoted epilepsyinduced neuronal death; caused abnormal neural axis, dendritic germination, and gliosis pathological changes; and shortened the latency of epileptic onset, thus promoting recurrent epilepsy [15]. More interestingly, treatment with celecoxib, an inhibitor of COX-2, restored pharmacosensitivity in a rat model of pharmacoresistant epilepsy by downregulating the expression of PGP, confirming that the COX-2/prostaglandin E2 (PGE2) inflammatory response pathway is closely related to MDT-mediated refractory epilepsy [16].

In Oriental societies, herbs and herbal preparations have been widely used as medication by consumers for many centuries to treat various diseases. Some herbal medications may be effective alternatives in the treatment of neuropsychiatric diseases, such as depression, anxiety, and insomnia. Chaihu plus Longgu Muli decoction (CLMD, saiko-ka-ryukotsu-borei-to in Japanese), a traditional Chinese medical compound, has been applied as a remedy for over 1000 years. Compositionally, this herbal formula is made of 12 indigenous components: Radix bupleuri, Ossa draconis, Radix scutellariae, Zingiberis rhizoma, red lead, Panax ginseng, Ramulus cinnamomi, Poria, Concha ostreae, Rhizoma pinelliae, Radix et rhizoma rhei, and Fructus ziziphi jujubae. (The composition and amount of each component as a percentage of the whole formula are shown in Table 1.) Recent studies have demonstrated that the use of CLMD in the treatment of neuropsychiatric disorders had very few side effects $[17,18]$. However, the efficacy and mechanism of CLMD therapy for epilepsy have not been reported internationally.
TABLE 1: Composition of Chaihu plus Longgu Muli decoction and the amount of each component as a percentage of the whole formula.

\begin{tabular}{lc}
\hline Medicinal component & Amount \\
\hline Radix bupleuri & $16.67 \%$ \\
Ossa draconis & $6.25 \%$ \\
Radix scutellariae & $6.25 \%$ \\
Zingiberis rhizoma & $6.25 \%$ \\
Red lead & $6.25 \%$ \\
Panax ginseng & $6.25 \%$ \\
Ramulus cinnamomi & $6.25 \%$ \\
Poria & $6.25 \%$ \\
Concha ostreae & $6.25 \%$ \\
Rhizoma pinelliae & $8.33 \%$ \\
Radix et rhizoma rhei & $8.33 \%$ \\
Fructus ziziphi jujubae & $16.67 \%$ \\
\hline
\end{tabular}

Kindling is a common experimental model for spontaneous and recurring seizures, and pentylenetetrazole (PTZ) is often used as a convulsing agent in experimental studies [19]. The kindling phenomenon is characterized by an increased susceptibility to epileptic seizures after repeated injections of PTZ [20]. Our previous study demonstrated that adenosine (Ade) had an inhibitory effect on the occurrence of epilepsy. Thus, we selected Ade A1 receptor- (AdeA1R-) knockout mice as the experimental subjects to increase susceptibility to epilepsy. In the current study, the effect of CLMD administration on epileptic seizures and brain injury was evaluated in PTZ-kindled mice, and the mechanism of CLMD action via the COX-2/PGE2/MDT pathway was investigated. We aimed to provide a theoretical basis for the clinical application of CLMD and open up new perspectives for the treatment of refractory epilepsy with integrated Chinese and Western medicine.

\section{Materials and Methods}

2.1. Chemicals and Reagents. PTZ (P6500) was purchased from Sigma-Aldrich (St. Louis, MO, USA). Hematoxylin (PAB1815), eosin (PAB1816), and Nissl's staining solutions (PAB180022) were purchased from Bioswamp (Wuhan, China). TRIzol reagent (15596-018), RevertAid First Strand cDNA Synthesis Kit (K1622), and SYBR ${ }^{\mathrm{TM}}$ Green PCR Master Mix (4364344) were purchased from Thermo Fisher Scientific (Waltham, MA, USA). Total protein extraction kit (W034), total protein bicinchoninic acid assay kit (A045-3), and enhanced chemiluminescence reagent (P0018A) were purchased from Nanjing Jiancheng Bioengineering Institute (Jiangsu, China). Rabbit primary antibodies against PGP (PAB18514, Bioswamp, Wuhan, China), MRP1 (PAB19878, Bioswamp, Wuhan, China), and fluorescein- (FITC-) conjugated AffiniPure goat anti-rabbit IgG (H+L) (PAB160016, Bioswamp, Wuhan, China) were purchased from Bioswamp. Rabbit primary antibodies against COX-2 (ab52237, Abcam, Cambridge, UK), PGE2 (ab167171, Abcam, Cambridge, UK), adenylate kinase (ADK, ab38010, Abcam, UK), GAPDH (ab37168, Abcam, Cambridge, UK), and goat anti-rabbit IgG (ab6721, Abcam, Cambridge, UK) 
were purchased from Abcam. Other materials with the highest analytical grade were purchased from Sinopharm Chemical Reagent Co., Ltd. (Shanghai, China).

2.2. Plant Material. The medicinal components used to prepare CLMD were provided by the Chinese Medicine Preparation Center, Wuhan First Hospital, and are listed in Table 1. CLMD was prepared by water boiling and alcohol extraction; mix all the drugs and soak for 30 minutes, then decoct on high flame for 20 minutes, then turn to slow fire for 30 minutes. The concentration of the crude drug in the obtained decoction was $1 \mathrm{~g} / \mathrm{ml}$.

2.3. Animals. Thirty adult male AdeA1R-knockout BALB/c mice weighing 20-25 g were purchased from Beijing Biocytogen Co., Ltd. (Beijing, China). The animals were housed in opaque polypropylene cages in a standard $12 \mathrm{~h} / 12 \mathrm{~h}$ light/dark cycle with food and water ad libitum at $22 \pm 2^{\circ} \mathrm{C}$ and $55-60 \%$ humidity. The mice were allowed to adapt to the above-mentioned conditions for seven days before experimentation. All animal experiments were approved by the Animal Ethics Committee of Wuhan First Hospital and complied with the Declaration of the National Institutes of Health Guide for Care and Use of Laboratory Animals (Publication No. 85-23, revised 1985).

2.4. Experimental Grouping and PTZ Kindling. The mice were divided into five groups ( $n=6$ per group): control (no kindling), model (PTZ-induced kindling), L-CLMD (low-dose CLMD followed by kindling), M-CLMD (medium-dose CLMD followed by kindling), and H-CLMD (high-dose CLMD followed by kindling). The mice in the L-CLMD, MCLMD, and H-CLMD group were administered CLMD at $5 \mathrm{ml} / \mathrm{kg}(\mathrm{mg} / \mathrm{kg}), 10 \mathrm{ml} / \mathrm{kg}(\mathrm{mg} / \mathrm{kg})$, and $20 \mathrm{ml} / \mathrm{kg}(\mathrm{mg} / \mathrm{kg})$, respectively, by gavage for one week before kindling. Mice in the control and model groups were administered $0.9 \%$ normal saline at $10 \mathrm{ml} / \mathrm{kg}(\mathrm{mg} / \mathrm{kg})$ body weight by gavage for one week.

To establish the epilepsy model, PTZ was dissolved in $0.9 \%$ saline and administered intraperitoneally (i.p.) with a subconvulsant dose of $35 \mathrm{mg} / \mathrm{kg}$ every day for 21 days. Mice in the control group were injected i.p. with the same amount of $0.9 \%$ saline. After daily PTZ injection, the convulsive behaviors of each mouse were observed, and the latency, sustained time, and grade/score of epileptic seizures were recorded. Epileptic seizures were scored using the following scale (according to Racine) [21]: 0, no response; 1, hyperactivity and vibrissae twitching; 2 , head nodding, head clonus, and myoclonic jerk; 3, unilateral forelimb clonus; 4, bilateral forelimb clonus with preservation of righting reflex; and 5, generalized tonic-clonic seizure with loss of righting reflex. The mouse was considered kindled if it exhibited a score of 4 or 5 on three consecutive injections of PTZ. As expected, the mice in the control group, which were challenged with saline, did not exhibit seizure and were not included in the behavioral analysis. The kindled mice from the model and CLMD-treated groups were subjected to behavioral analysis for 21 days.

2.5. Sample Collection. Mice were sacrificed by decapitation after anesthetized with $1 \%$ pentobarbital sodium $(50 \mathrm{mg} / \mathrm{kg})$ at the end of the observation period of 21 days, followed by immediate removal of the two hemispheres of the brain. The cortex and hippocampus from one hemisphere were fixed for $24 \mathrm{~h}$ in $10 \%$ paraformaldehyde solution for subsequent tissue staining. The cortex and hippocampus from the other hemisphere were frozen at $-80^{\circ} \mathrm{C}$ for other molecular experiments.

2.6. Hematoxylin and Eosin (HE) Staining. The cortex and hippocampus were chosen since they are the most sensitive areas to toxic insults and are important for maintaining epileptic seizures. Portions of the cortex and hippocampus were fixed in $10 \%$ paraformaldehyde solution for at least $24 \mathrm{~h}$, dehydrated with a sequence of ethanol solutions, and processed for paraffin embedding. Sections of $5 \mu \mathrm{m}$ in thickness were cut, deparaffinized, rehydrated, stained with $\mathrm{HE}$, and examined under a light microscope (Olympus, Tokyo, Japan).

2.7. Nissl's Staining. Portions of the cortex and hippocampus were fixed in $10 \%$ paraformaldehyde solution for at least $24 \mathrm{~h}$. Thereafter, they were dehydrated, paraffin-embedded, and sliced. Sections of $5 \mu \mathrm{m}$ in thickness were stained with Nissl's staining solution for $10 \mathrm{~min}$ at $37^{\circ} \mathrm{C}$, dehydrated in $95 \%$ ethanol for $2 \mathrm{~min}$, and rendered transparent in xylene for $5 \mathrm{~min}$. The sections were observed under a light microscope (Olympus, Tokyo, Japan).

2.8. Immunofluorescence. As previously mentioned, $5 \mu \mathrm{m}$ thick paraffin sections of the cortex and hippocampus were prepared. The sections were processed by baking, dewaxing, antigen repair, and endogenous enzyme removal. Then, the sections were blocked in donkey serum and incubated with primary antibodies against PGP $(1: 200)$ and MRP1 $(1: 200)$ at $4^{\circ} \mathrm{C}$ overnight. Then, the sections were incubated with FITC-conjugated AffiniPure goat anti-rabbit IgG second antibody $(1: 500)$ for $1 \mathrm{~h}$ at $37^{\circ} \mathrm{C}$, and the nuclei were stained with $4^{\prime}, 6$-diamidino-2-phenylindole for $10 \mathrm{~min}$ at $37^{\circ} \mathrm{C}$. The sections were observed under a fluorescence microscope (Olympus, Tokyo, Japan).

2.9. Quantitative Reverse Transcription Polymerase Chain Reaction ( $q R T-P C R)$. Frozen tissues from the cortex and hippocampus were ground in liquid nitrogen. Total RNA was extracted from tissues using TRIzol reagent and reverse-transcribed into cDNA using the RevertAid First Strand cDNA Synthesis Kit according to the manufacturer's instructions. qRT-PCR was performed in a 20- $\mu$ l reaction mixture containing SYBR ${ }^{\mathrm{TM}}$ Green PCR Master Mix, cDNA, and each primer at $0.2 \mathrm{mmol} / 1$ at $95^{\circ} \mathrm{C}$ for $10 \mathrm{~min}, 40$ cycles at $95^{\circ} \mathrm{C}$ for $10 \mathrm{~s}$, and $60^{\circ} \mathrm{C}$ for $45 \mathrm{~s}$. Data were collected using the QuantStudio ${ }^{\text {TM }} 6$ Flex Real-Time PCR System (Applied Biosystems). The relative amount of each gene was normalized to the housekeeping gene GAPDH. Relative quantity values were analyzed using the $2^{-\Delta \Delta \mathrm{Ct}}$ method. The primer sequences are shown as follows: PGP F, $5^{\prime}$-CCTGGGCTC CACCTGTA-3', R, 5'-GGACCATTTTCTTCTTG-3'; MRP1 F, 5' -CCTCAACAAAACCAAAA-3', R, 5' -GGGC TGACCAGTAACAC- $3^{\prime}$; and GAPDH F, $5^{\prime}$-CCTTCCGTG TTCCTAC-3', R, 5' -GACAACCTGGTCCTCA-3'. 
2.10. Protein Extraction and Western Blot. Proteins were extracted from the cortex and hippocampus using a total protein extraction kit and quantified by the bicinchoninic acid assay kit. Whole proteins were electrophoresed in 10-15\% polyacrylamide gels, and the separated proteins were transferred to polyvinylidene difluoride membranes. Nonspecific binding was blocked with $5 \%$ skimmed milk for $2 \mathrm{~h}$ at $37^{\circ} \mathrm{C}$. The membranes were then incubated with rabbit antibodies against COX-2 (1:1000), PGE2 $(1: 5000)$, and ADK $(1: 500)$ at $4{ }^{\circ} \mathrm{C}$ overnight with GAPDH $(1: 1000)$ as a control. Next, the membranes were incubated with goat anti-rabbit IgG $(1: 5000)$ for $1 \mathrm{~h}$ at room temperature. Images were acquired using a multifunctional gel imaging system (Image Quant LAS 500, General Electric, Fairfield, CT, USA) after incubation with enhanced chemiluminescence reagent. GAPDH act as the internal reference, relative expression of target protein $=$ target protein/GAPDH.

2.11. Statistical Analyses. All values are presented as the mean \pm standard deviation (SD). One-way analysis of variance followed by the Tukey test was performed to compare differences between multiple groups (more than two) using SPSS 19.0 software (IBM Corp., Armonk, NY, USA). $P<0.05$ is considered statistically significant.

\section{Results}

3.1. CLMD Reduced the Susceptibility to and Activity of Epileptic Seizures. No mouse died after the 21-day behavioral analysis, and the control mice challenged with saline did not exhibit seizures. However, the mice in the PTZ-induced and CLMD treatment groups showed varying degrees of seizures. In mice subjected to PTZ injection, the seizure grade on days 19,20 , and 21 was 4 or 5 , confirming that the mice were successfully kindled by PZT. On day 21, the mean latency of epileptic seizures was significantly increased in the CLMDtreated mice compared with that in the nontreated kindled mice, especially at high-dose CLMD $(P<0.01$, Figure $1(\mathrm{a}))$. In addition, high-dose CLMD led to significant reductions in sustained time and grade of epileptic seizures $(P<0.05$, $P<0.01$ ), while medium- and low-dose CLMD induced no significant change (Figures $1(\mathrm{~b})$ and $1(\mathrm{c})$ ). These results indicated that CLMD, especially at a high dose, slowed the time and decreased the grade of epileptic seizures.

3.2. CLMD Alleviated Brain Damage in Epileptic Mice. HE staining showed that the neurons in the cortex and hippocampus in the control group were well arranged with a normal morphology. With PTZ-induced kindling, the neurons became randomly arranged, and some contained obvious vacuoles in the cytoplasm whereas some were swollen and unevenly distributed. Nuclear pyknosis and hyperchromia occurred, along with the disappearance of nucleolus. In the CLMD-treated neurons, the increase in CLMD concentration induced the scattered neurons to gradually reorganize into a normal arrangement. Symptoms such as swelling of the neuron cell body and hyperchromatism of nuclear pyknosis were further alleviated, especially at high-dose CLMD (Figure 2(a)). Nissl's staining showed that neurons in the cortex and hippocampus of the control group were arranged in an orderly manner, and the cytoplasm was rich in Nissl bodies. Neurons in the PTZ-kindled mice showed a decrease in the number of Nissl bodies in the cortex and hippocampus. Some cells were swollen, with fuzzy cell contour and incomplete morphology. In CLMD-treated mice, with the increase in CLMD concentration, the number of Nissl bodies increased gradually. Neuron swelling gradually disappeared, and the cell morphology tended to be normal, especially at high-dose CLMD (Figure 2(b)). These results demonstrated that CLMD reduced brain damage in epileptic mice, as reflected in the increased number of surviving neurons in the cortex and hippocampus, especially at highdose CLMD.

3.3. CLMD Decreased the $m R N A$ and Protein Levels of PGP and MRP1 in the Cortex and Hippocampus of Epileptic Mice. In the PTZ-kindled mice, the mRNA levels of PGP and MRP1 were significantly increased compared to those in the control group $(P<0.01)$ in both the cortex (Figure 3(a)) and hippocampus (Figure 3(b)). CLMD at various doses reduced the mRNA levels of PGP and MRP1 to varying degrees $(P<0.01)$, and the effect was most prominently observed at high-dose CLMD. Similar to mRNA expression, immunofluorescence showed that after PTZ kindling, the protein levels of PGP and MRP1 (green fluorescence) in both the cortex (Figure 4(a)) and hippocampus (Figure 4(b)) were significantly higher than those in the control group. CLMD at different doses reduced the protein levels of PGP and MRP1 to various extents, and the effect was especially noticeable at high-dose CLMD. The decreased in the protein levels of PGP and MRP1 in the cortex and hippocampus in epileptic mice demonstrated here may encourage CLMD to reach the lesion site and facilitate its therapeutic effect.

3.4. CLMD Played an Antiepileptic Role by Downregulating COX-2/PGE2/ADK Signaling. In PTZ-kindled mice, the protein levels of COX-2, PGE2, and ADK in both the cortex (Figures 5(a) and 5(b)) and hippocampus (Figures 5(c) and $5(d))$ were significantly increased compared with those in the control group $(P<0.01)$. CLMD reduced the protein levels of COX-2, PGE2, and ADK to varying degrees, and its effect was more remarkable at high dose than at low and medium dose $(P<0.01)$. These observations implied that the COX-2/PGE2/ADK pathway may be involved in the antiepileptic action of CLMD.

\section{Discussion}

The present study revealed the therapeutic potential of CLMD on PTZ-kindled epileptic mice. PTZ-induced kindling is a well-accepted model to investigate and analyze the pathophysiological mechanisms and behavioral characteristics during epileptogenesis [22]. The symptoms of epileptic seizures induced by PTZ kindling in animal models were found to be clinically relevant and similar to those of human temporal lobe epilepsy [23] and generalized tonic-clonic convulsions [22]. In the present study, administration of PTZ $(35 \mathrm{mg} / \mathrm{kg})$ for a period of 21 days (21 injections) in AdeA1R-knockout mice 


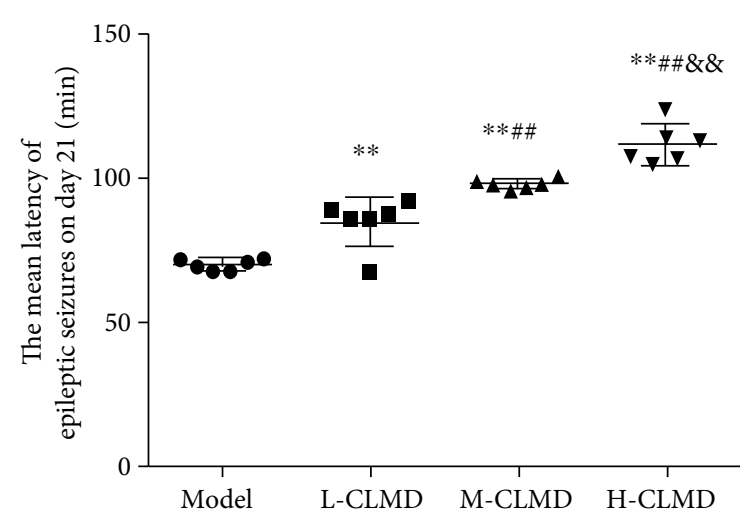

(a)

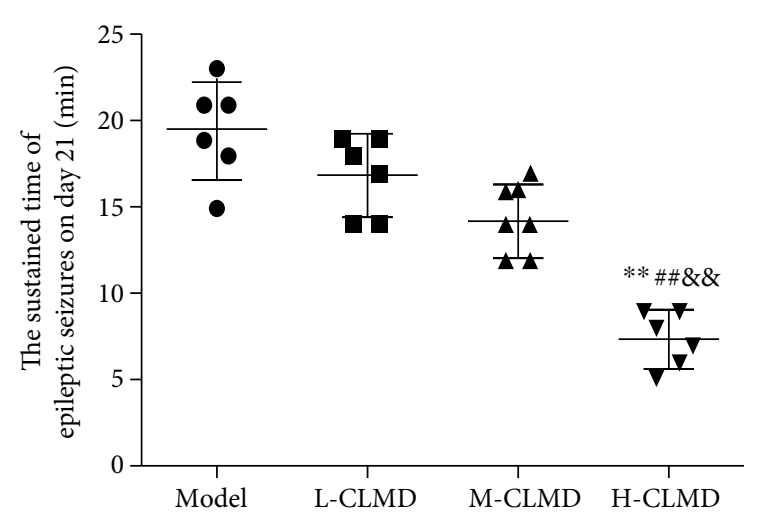

(b)

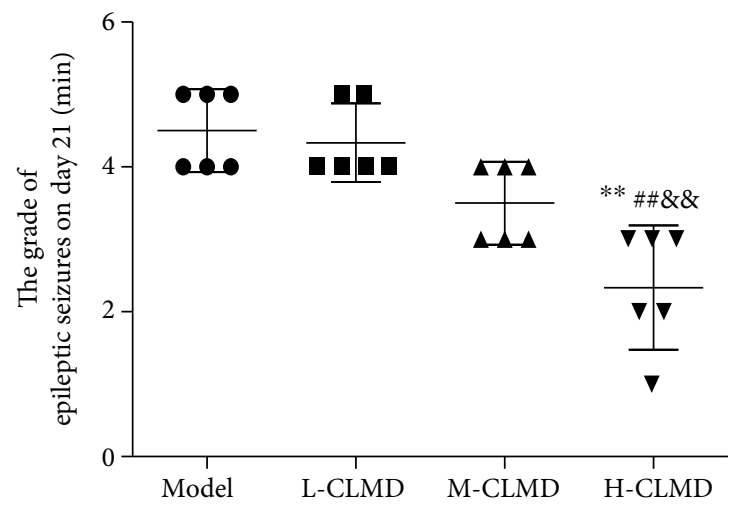

(c)

FIgURE 1: Indices of behavioral analysis in mice after 21 days of observation: (a) mean latency of epileptic seizures; (b) sustained time of epileptic seizures; (c) grade of epileptic seizures. ${ }^{* *} P<0.01$ vs. model; ${ }^{\# \#} P<0.01$ vs. L-CLMD; ${ }^{\&} P<0.05$ and ${ }^{8 \&} P<0.01$ vs. M-CLMD. L: low dose; M: medium dose; H: high dose; CLMD: Chaihu plus Longgu Muli decoction.

successfully established the epilepsy model. In turn, high-dose CLMD $(20 \mathrm{ml} / \mathrm{kg}(\mathrm{mg} / \mathrm{kg}))$ significantly attenuated the susceptibility and activity of PTZ-induced epileptic seizures in mice, indicating its antiepileptogenic potential. In addition, epilepsy is known to cause various behavioral phenomena, including anxiety, depression, and learning and memory deficit [24].

CLMD was originally recorded in the "Treatise on Febrile Disease," which has a history of nearly two thousand years and was written by Zhang Zhongjing, an expert in medicine and pharmacy in the Eastern Han dynasty in Chinese history. In traditional Chinese medicine, CLMD has been extensively used to treat neuropsychiatric disorders such as depressive illness. In a rat model of unpredictable chronic mild stress, total saponins extracted from Chaihu-jia-longgu-muli-tang reversed depression-like behaviors, restored the reduced levels of monoamine neurotransmitters, and upregulated the expression of brain-derived neurotrophic factor [25]. Meanwhile, saponins extracted from Chaihu-jia-longgumuli-tang showed significant in vitro neuroprotective action against corticosterone-induced lesions in PC12 cells, a rat pheochromocytoma cell line [18]. Since depression is a complication of epilepsy, previous studies have laid a theoretical foundation for our experimental hypothesis that the CLMD might also have antiepileptic effects.

Many studies have reported that repeated injection of subconvulsive doses of PTZ increased the susceptibility of experimental animals, resulting in a fully kindled state [26, 27]. In this case, brief epileptic seizures last for several minutes and resemble human epilepsy, along with histopathological abnormalities in some regions of the brain such as the cortex and hippocampus [28]. Extensive studies in experimental models have reported that continuous epileptic seizures or even brief repetitive seizures caused by kindling provoked pathological neurodegeneration in the brain [29, 30]. In our study, pathological assessment indicated that the PTZ-kindled mice showed typical neurofunctional degeneration in cortical and hippocampal tissues. As expected, CLMD alleviated brain injury in kindled mice. In particular, it increased the number of surviving neurons in the cortex and hippocampus, which may be directly related to the short onset time and low grade of epileptic seizures. However, amelioration of seizure activity does not necessarily imply the direct anticonvulsant property of CLMD. Therefore, we followed up on the study by focusing on the cortex and hippocampus to explore the in-depth mechanism of CLMD.

In recent years, drug resistance has become an obstacle in the treatment of refractory epilepsy. Drug resistance is mainly caused by the maladjustment of the expression of MDTs, such as PGP and MRP1 [31]. An imaging study showed that refractory epilepsy was associated with localized overexpression of drug transporter proteins such as PGP in the epileptic focal region, which actively extruded AEDs from 

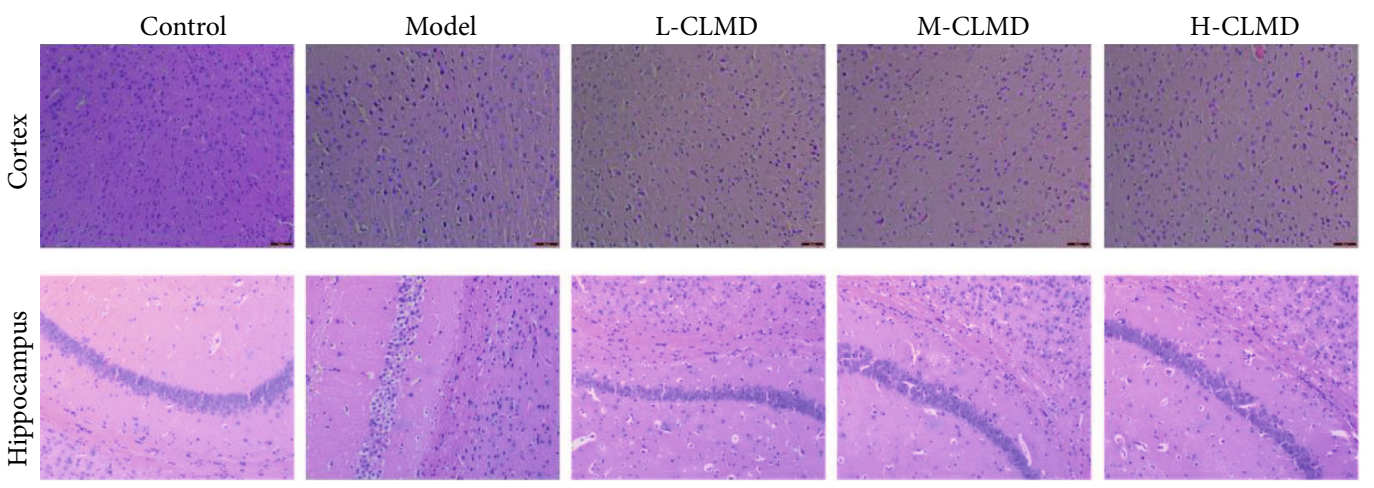

HE staining $(\times 200)$

(a)
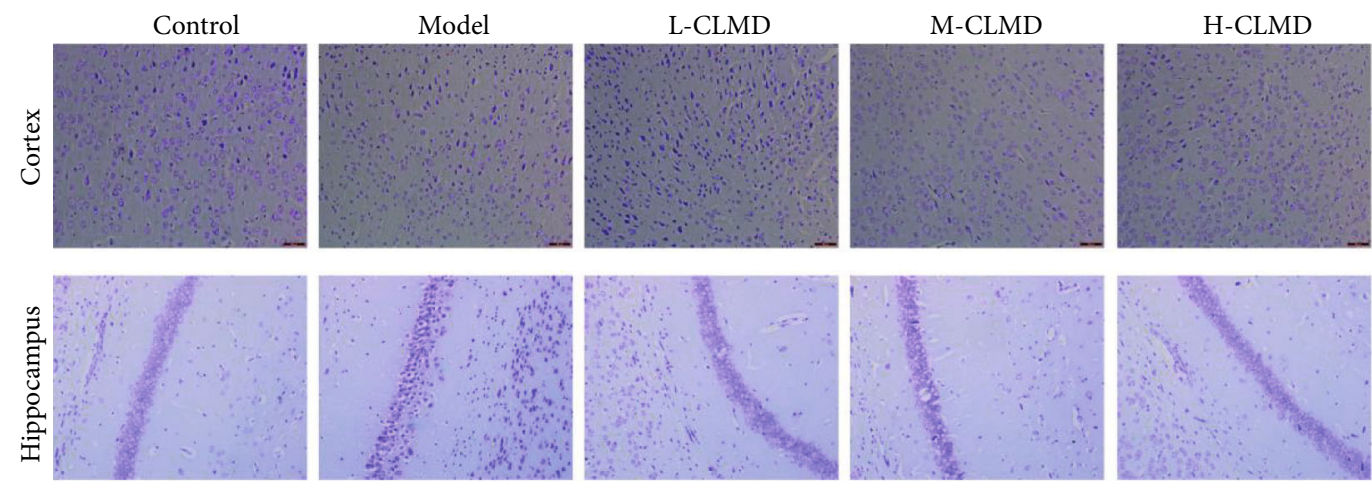

Nissl's staining $(\times 200)$

(b)

FIgURE 2: Histopathological evaluation of brain damage in the cortex and hippocampus of PTZ-kindled epileptic mice. (a) HE staining ( $\times 200)$ and (b) Nissl's staining of the cortex and hippocampus (×200). L: low dose; M: medium dose; H: high dose; CLMD: Chaihu plus Longgu Muli decoction.

their intended site of action [32]. Meanwhile, a metaanalysis of nine studies revealed that neural overexpression of MRP1 was closely related to refractory epilepsy [33]. Furthermore, Potschka and Loscher found that when PGP and MRPs were overexpressed, the concentration of AEDs in cells was decreased in the lesion area [34]. Tishler et al. measured the concentration of phenytoin in the brain tissue of patients with refractory epilepsy after surgical resection and found that the concentration of phenytoin in PGP-positive cells was only $25 \%$ of that in PGPnegative cells [35]. Cox et al. showed that the artificial regulation of PGP and MRP2 expression altered the pharmacodynamic and histological distribution of certain AEDs [36]. In the current study, we found that CLMD significantly reduced the expression of PGP and MRP1 in cortical and hippocampal tissues of kindled mice. The findings suggested that suppressing the expression of MDTs, such as PGP and MRP1, is one of the mechanisms by which CLMD exerts antiepileptic effects.

Ade is an endogenous anticonvulsive neuromodulator with neuroprotective properties, and the lack of Ade can directly lead to the aggravation of epileptic seizures, which is difficult to control and can cause persistent epilepsy [37]. Ade is mostly phosphorylated by ADK into adeno- sine monophosphate, adenosine diphosphate, and ATP to complete the recycling of Ade [38]. MDTs are a superfamily of ATP-binding proteins, and the overexpression of MDTs can induce the decomposition of a large amount of ATP, resulting in Ade deficiency. Thus, the Ade system is closely related to refractory epilepsy mediated by MDT overexpression. An interesting study showed that ADK overexpression in astrocytes triggered seizures [39]. On the contrary, antisense-mediated knockdown of ADK expression within astrocytes inhibited spontaneous seizures [40]. Similarly, Gouder et al. found that the overexpression of ADK in epileptic hippocampus contributed to epileptogenesis, while seizures and interictal spikes were suppressed by the injection of low-dose 5-iodotubercidin, an inhibitor of ADK [41]. In our study, the trend of ADK expression was consistent with that of MDTs (PGP and MRP1), and CLMD administration significantly reduced their expression. Thus, we confirm that the antiepileptic effect of CLMD is related to the downregulation of ADK expression.

It is well known that epileptic seizures result in and enhance brain damage and related processes such as inflammation. COXs are rate-limiting enzymes in metabolic pathways in which arachidonic acid is converted into PGs. 


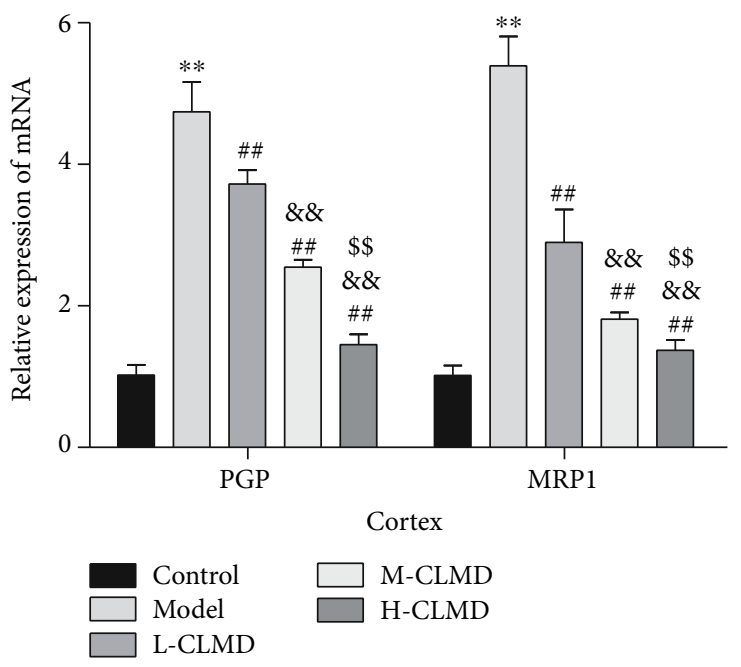

(a)

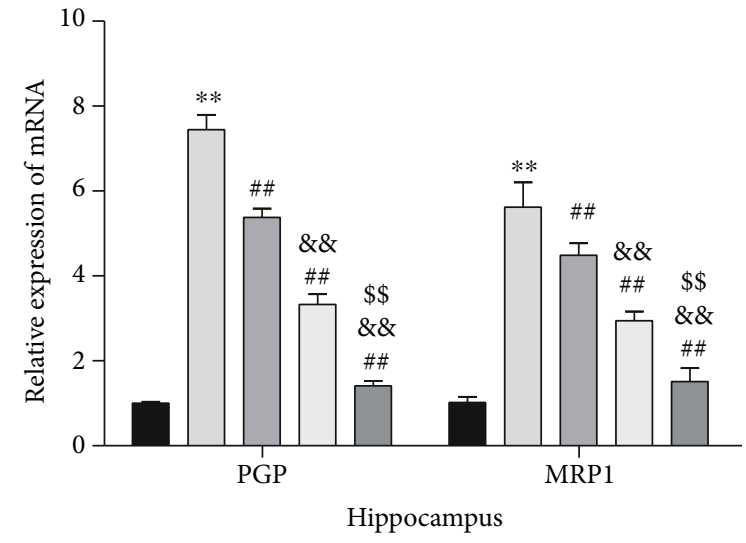

(b)

FIGURE 3: mRNA levels of PGP and MRP1 in the cortex and hippocampus of PTZ-kindled epileptic mice. mRNA levels of PGP and MRP1 in the (a) cortex and (b) hippocampus. ${ }^{* *} P<0.01$ vs. control; ${ }^{\# \#} P<0.01$ vs. L-CLMD; ${ }^{\& \&} P<0.01$ vs. L-CLMD; ${ }^{\$ \$} P<0.01$ vs. M-CLMD. L: low dose; M: medium dose; H: high dose; CLMD: Chaihu plus Longgu Muli decoction; PGP: p-glycoprotein; MRP1: multidrug resistanceassociated protein 1 .
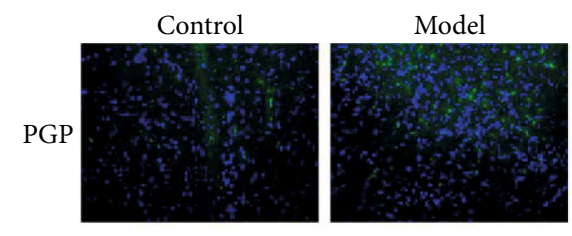

L-CLMD
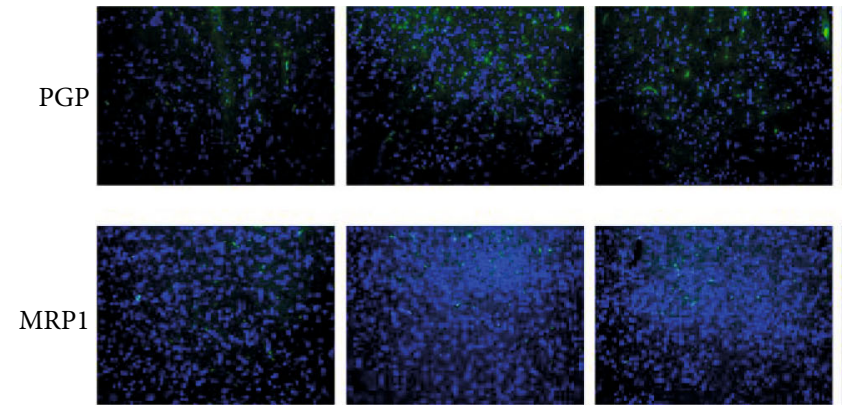

Cortex IF $(\times 200)$

(a)

Hippocampus IF $(\times 200)$

(b)
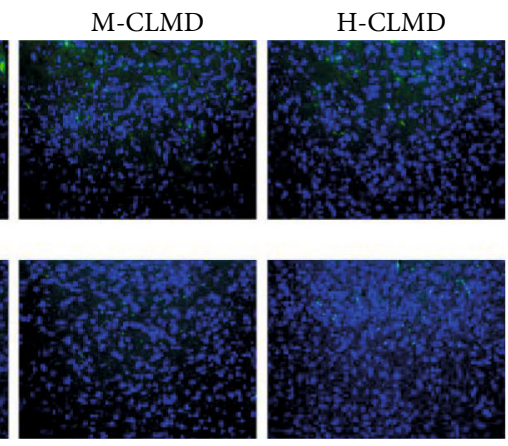

M-CLMD
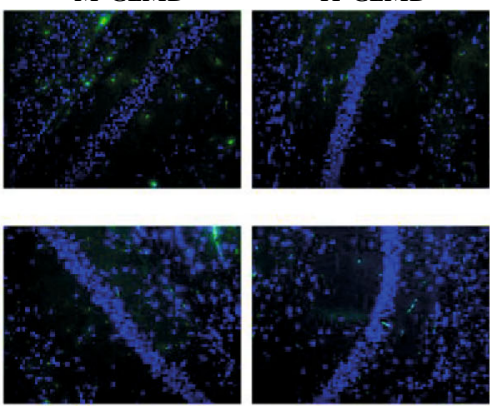

H-CLMD

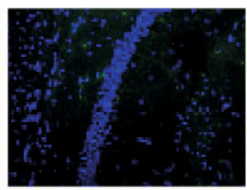

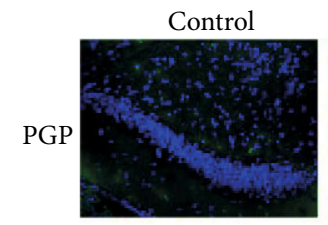

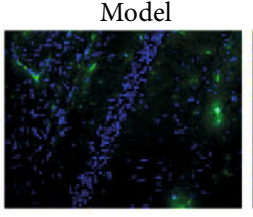

L-CLMD
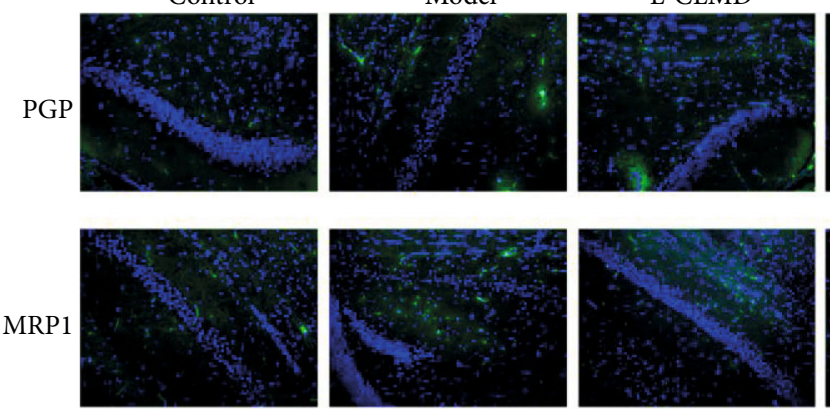

FIGURE 4: Immunofluorescence evaluation of PGP and MRP1 expression in the cortex and hippocampus of PTZ-kindled epileptic mice. Immunofluorescence staining of PGP and MRP1 in the (a) cortex $(\times 200)$ and (b) hippocampus $(\times 200)$. L: low dose; M: medium dose; H: high dose; CLMD: Chaihu plus Longgu Muli decoction; PGP: p-glycoprotein; MRP1: multidrug resistance-associated protein 1. 

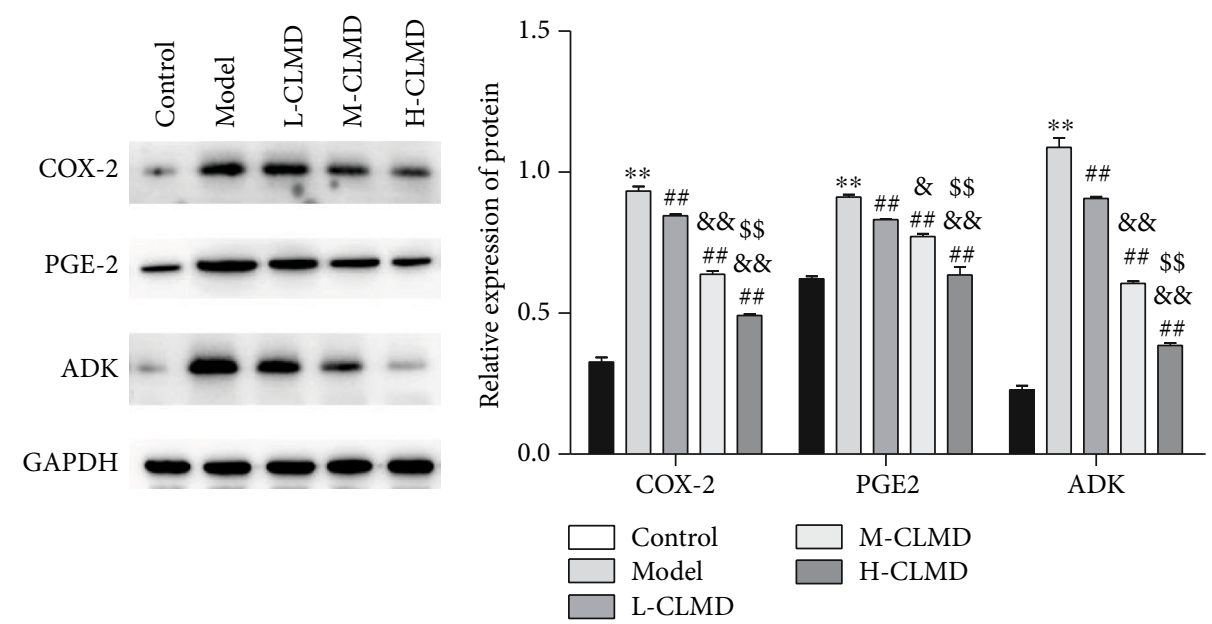

(a)

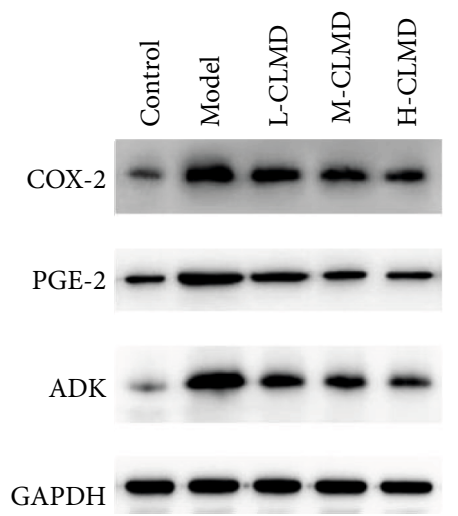

(c) (b)

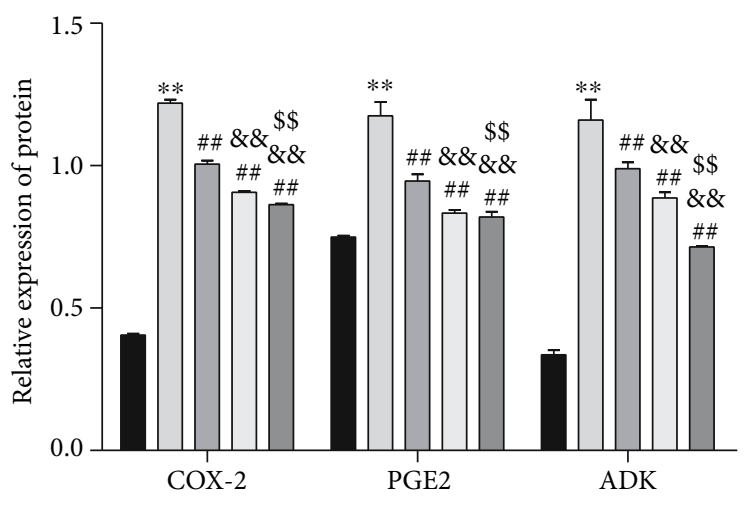

(d)

FIGURE 5: Evaluation of the expression of proteins involved in the COX-2/PGE2/ADK pathway in the cortex and hippocampus of PTZkindled epileptic mice. (a) Western blot and (b) quantitative analysis of the protein levels of COX-2, PGE2, and ADK in the cortex. (c) Western blot and (d) quantitative analysis of the protein levels of COX-2, PGE2, and ADK in the hippocampus. ${ }^{* *} P<0.01$ vs. control; ${ }^{\#} P<0.05,{ }^{\# \#} P<0.01$ vs. model; ${ }^{\&} P<0.5,{ }^{8 \&} P<0.01$ vs. L-CLMD; ${ }^{\$ \$} P<0.01$ vs. M-CLMD. L: low dose; M: medium dose; H: high dose; CLMD: Chaihu plus Longgu Muli decoction; COX-2: cyclooxygenase-2; PGE2: prostaglandin E2; ADK: adenylate kinase; GAPDH: glyceraldehyde-phosphate dehydrogenase.

COX-2 is the isoform induced at injury/inflammation sites and is expressed constitutively in several tissues, such as tissues in the central nervous system, and is involved in neurodegenerative diseases associated with increased excitatory activity[42]. Not only patients with temporal lobe epilepsy [43] but also genetically seizure-susceptible mice [44] exhibited increased COX-2 expression in the brain, suggesting that COX-2 may have critical functions in convulsive states. However, in experiments performed in rats, the specific COX-2 inhibitor nimesulide was shown to reduce white matter damage in chronic cerebral ischemia, limit kainateinduced oxidative damage in the hippocampus, and alleviate motor and cognitive dysfunction following diffuse traumatic brain injury [45]. In a rat model of absence epilepsy, the selective COX-2 inhibitor etoricoxib exerted antiepileptogenic effects by reducing the synthesis of PGs and suppressing neuroinflammation-dependent adaptive processes [46]. The inhibition of PGE2, a key mediator in COX-2 signaling, by specific antibodies or COX-2 inhibitor celecoxib resulted in decreased PTZ susceptibility [47, 48]. Oliveira et al. observed the electroencephalogram changes in rats injected with PTZ by administering antibodies against PGE2 and found that the antibodies reduced PTZ-induced epileptic seizures [42]. In our study, we observed a decrease in COX2 and PGE2 protein expression during CLMD therapy. We conclude that CLMD exerted antiepileptic effects by downregulating COX-2/PGE2 signaling.

\section{Conclusion}

We suggest that CLMD acts as a dose-dependent antiepileptic agent. The therapeutic effects of CLMD are mainly related to the reduction in seizure time and grade, repair of brain damage, and increase in the number of surviving neurons. These effects might be due to COX-dependent mechanisms such as reduced synthesis of PGE2 and the resulting attenuation of neuroinflammatory injury. Furthermore, the involvement of other mechanisms such as the downregulation of MDTs and 
consequent downregulation of ADK should be considered. There are indeed shortcomings in this study, and further experiments are needed to clarify the relationship between CLMD and COX-2 or MDT inhibitors to support the antiepileptic mechanisms of CLMD.

\section{Data Availability}

The data used to support the findings of this study are available from the corresponding author upon request.

\section{Conflicts of Interest}

There are no conflicts of interest.

\section{Authors' Contributions}

Ping Shan and Jilong Zhang contributed equally.

\section{Acknowledgments}

This work was supported by the grant from the National Natural Science Foundation of China (Grant No. 81703870).

\section{References}

[1] M. R. Keezer, S. M. Sisodiya, and J. W. Sander, "Comorbidities of epilepsy: current concepts and future perspectives," The Lancet. Neurology, vol. 15, no. 1, pp. 106-115, 2016.

[2] A. Singh and S. Trevick, "The epidemiology of global epilepsy," Neurologic Clinics, vol. 34, no. 4, pp. 837-847, 2016.

[3] S. D. Shorvon, "The epidemiology and treatment of chronic and refractory epilepsy," Epilepsia, vol. 37, Suppl 2, pp. S1S3, 1996.

[4] S. Sinha and K. A. Siddiqui, "Definition of intractable epilepsy," Neurosciences, vol. 16, no. 1, pp. 3-9, 2011.

[5] D. S. Miller, "Regulation of P-glycoprotein and other ABC drug transporters at the blood-brain barrier," Trends in Pharmacological Sciences, vol. 31, no. 6, pp. 246-254, 2010.

[6] R. Kovacs, C. Raue, S. Gabriel, and U. Heinemann, "Functional test of multidrug transporter activity in hippocampalneocortical brain slices from epileptic patients," Journal of Neuroscience Methods, vol. 200, no. 2, pp. 164-172, 2011.

[7] S. Baltes, A. M. Gastens, M. Fedrowitz, H. Potschka, V. Kaever, and W. Loscher, "Differences in the transport of the antiepileptic drugs phenytoin, levetiracetam and carbamazepine by human and mouse P-glycoprotein," Neuropharmacology, vol. 52, no. 2, pp. 333-346, 2007.

[8] A. Lazarowski, L. Czornyj, F. Lubienieki, E. Girardi, S. Vazquez, and C. D'Giano, "ABC transporters during epilepsy and mechanisms underlying multidrug resistance in refractory epilepsy," Epilepsia, vol. 48, Suppl 5, pp. 140-149, 2007.

[9] L. Librizzi, F. Noe, A. Vezzani, M. de Curtis, and T. Ravizza, "Seizure-induced brain-borne inflammation sustains seizure recurrence and blood-brain barrier damage," Annals of Neurology, vol. 72, no. 1, pp. 82-90, 2012.

[10] A. Vezzani, D. Moneta, C. Richichi et al., "Functional role of inflammatory cytokines and antiinflammatory molecules in seizures and epileptogenesis," Epilepsia, vol. 43, Suppl 5, pp. 30-35, 2002.
[11] F. E. Dudek, "Mechanisms of seizure-induced inflammation of the brain: many possible roles for neuronal COX-2," Epilepsy Currents, vol. 12, pp. 115-117, 2012.

[12] M. G. Salvadori, C. R. Bandero, A. C. Jesse et al., "Prostaglandin E(2) potentiates methylmalonate-induced seizures," Epilepsia, vol. 53, no. 1, pp. 189-198, 2012.

[13] S. Keros and C. J. McBain, "Arachidonic acid inhibits transient potassium currents and broadens action potentials during electrographic seizures in hippocampal pyramidal and inhibitory interneurons," The Journal of neuroscience : the official journal of the Society for Neuroscience, vol. 17, no. 10, pp. 3476-3487, 1997.

[14] P. Sancho, P. Martin-Sanz, and I. Fabregat, "Reciprocal regulation of NADPH oxidases and the cyclooxygenase-2 pathway," Free Radical Biology \& Medicine, vol. 51, no. 9, pp. 1789-1798, 2011.

[15] J. T. Jarvela, F. R. Lopez-Picon, and I. E. Holopainen, "Agedependent cyclooxygenase- 2 induction and neuronal damage after status epilepticus in the postnatal rat hippocampus," Epilepsia, vol. 49, no. 5, pp. 832-841, 2008.

[16] J. Schlichtiger, A. Pekcec, H. Bartmann et al., "Celecoxib treatment restores pharmacosensitivity in a rat model of pharmacoresistant epilepsy," British Journal of Pharmacology, vol. 160, no. 5, pp. 1062-1071, 2010.

[17] T. Satoh, T. Takahashi, K. Iwasaki et al., "Traditional Chinese medicine on four patients with Huntington's disease," Movement Disorders, vol. 24, no. 3, pp. 453-455, 2009.

[18] W. Zhu, S. Ma, R. Qu, and D. Kang, “Antidepressant-like effect of saponins extracted from Chaihu-jia-longgu-muli-tang and its possible mechanism," Life Sciences, vol. 79, no. 8, pp. 749756, 2006.

[19] G. L. Holmes, A. Chronopoulos, C. E. Stafstrom et al., "Effects of kindling on subsequent learning, memory, behavior, and seizure susceptibility," Brain Research. Developmental Brain Research, vol. 73, no. 1, pp. 71-77, 1993.

[20] J. Ono, R. F. Vieth, and P. D. Walson, "Electrocorticographical observation of seizures induced by pentylenetetrazol (PTZ) injection in rats," Functional Neurology, vol. 5, no. 4, pp. 345-352, 1990.

[21] R. J. Racine, "Modification of seizure activity by electrical stimulation: II. Motor seizure," Electroencephalography and Clinical Neurophysiology, vol. 32, no. 3, pp. 281-294, 1972.

[22] A. Dhir, "Pentylenetetrazol (PTZ) kindling model of epilepsy," Current Protocols in Neuroscience, vol. 58, no. 1, 2012.

[23] M. G. Genkova-Papazova, B. Petkova, N. Shishkova, and M. Lazarova-Bakarova, "The GABA-B antagonist CGP 36742 prevent PTZ-kindling-provoked amnesia in rats," European Neuropsychopharmacology, vol. 10, no. 4, pp. 273-278, 2000.

[24] N. Geschwind, "Interictal behavioral changes in epilepsy," Epilepsia, vol. 24, Suppl 1, pp. S23-S30, 1983.

[25] L. F. Li, J. Lu, X. M. Li et al., "Antidepressant-like effects of the saponins extracted from Chaihu-jia-longgu-muli-tang in a rat unpredictable chronic mild stress model," Fitoterapia, vol. 83, no. 1, pp. 93-103, 2012.

[26] Y. Li, W. Y. Huang, C. Y. Lv et al., "Satb2 ablation decreases PTZ-induced seizure susceptibility and pyramidal neuronal excitability,” Brain Research, vol. 1695, pp. 102-107, 2018.

[27] C. R. Mason and R. M. Cooper, "A permanent change in convulsive threshold in normal and brain-damaged rats with repeated small doses of pentylenetetrazol," Epilepsia, vol. 13, no. 5, pp. 663-674, 1972. 
[28] L. F. Silva, M. S. Hoffmann, R. Gerbatin Rda et al., "Treadmill exercise protects against pentylenetetrazol-induced seizures and oxidative stress after traumatic brain injury," Journal of Neurotrauma, vol. 30, no. 14, pp. 1278-1287, 2013.

[29] M. I. Naseer, I. Ullah, M. H. Al-Qahtani et al., "Decreased GABABR expression and increased neuronal cell death in developing rat brain after PTZ-induced seizure," Neurological Sciences, vol. 34, no. 4, pp. 497-503, 2013.

[30] V. Sharma, B. Nehru, A. Munshi, and A. Jyothy, "Antioxidant potential of curcumin against oxidative insult induced by pentylenetetrazol in epileptic rats," Methods and Findings in Experimental and Clinical Pharmacology, vol. 32, no. 4, pp. 227-232, 2010.

[31] S. M. Sisodiya, W. R. Lin, B. N. Harding, M. V. Squier, and M. Thom, "Drug resistance in epilepsy: expression of drug resistance proteins in common causes of refractory epilepsy," Brain, vol. 125, no. 1, pp. 22-31, 2002.

[32] W. Loscher and O. Langer, "Imaging of P-glycoprotein function and expression to elucidate mechanisms of pharmacoresistance in epilepsy," Current Topics in Medicinal Chemistry, vol. 10, no. 17, pp. 1785-1791, 2010.

[33] Y. Sun, X. Luo, K. Yang et al., "Neural overexpression of multidrug resistance-associated protein 1 and refractory epilepsy: a meta-analysis of nine studies," The International Journal of Neuroscience, vol. 126, pp. 308-317, 2016.

[34] H. Potschka and W. Loscher, "In vivo evidence for Pglycoprotein-mediated transport of phenytoin at the bloodbrain barrier of rats," Epilepsia, vol. 42, no. 10, pp. 12311240, 2001.

[35] D. M. Tishler, K. I. Weinberg, D. R. Hinton, N. Barbaro, G. M. Annett, and C. Raffel, "MDR1 gene expression in brain of patients with medically intractable epilepsy," Epilepsia, vol. 36, no. 1, pp. 1-6, 1995.

[36] D. S. Cox, K. R. Scott, H. Gao, and N. D. Eddington, "Effect of P-glycoprotein on the pharmacokinetics and tissue distribution of enaminone anticonvulsants: analysis by population and physiological approaches," The Journal of Pharmacology and Experimental Therapeutics, vol. 302, no. 3, pp. 10961104, 2002.

[37] E. Avsar and R. M. Empson, “Adenosine acting via A1 receptors, controls the transition to status epilepticus-like behaviour in an in vitro model of epilepsy," Neuropharmacology, vol. 47, no. 3, pp. 427-437, 2004.

[38] A. C. Newby, "The role of adenosine kinase in regulating adenosine concentration," The Biochemical Journal, vol. 226, no. 1, pp. 343-344, 1985.

[39] H. Y. Shen, H. Sun, M. M. Hanthorn et al., "Overexpression of adenosine kinase in cortical astrocytes and focal neocortical epilepsy in mice," Journal of Neurosurgery, vol. 120, no. 3, pp. 628-638, 2014.

[40] P. Theofilas, S. Brar, K. A. Stewart et al., "Adenosine kinase as a target for therapeutic antisense strategies in epilepsy," Epilepsia, vol. 52, no. 3, pp. 589-601, 2011.

[41] N. Gouder, L. Scheurer, J. M. Fritschy, and D. Boison, “Overexpression of adenosine kinase in epileptic hippocampus contributes to epileptogenesis," The Journal of neuroscience : the official journal of the Society for Neuroscience, vol. 24, no. 3, pp. 692-701, 2004.

[42] M. S. Oliveira, A. F. Furian, L. F. Royes et al., "Cyclooxygenase2/PGE2 pathway facilitates pentylenetetrazol-induced seizures," Epilepsy Research, vol. 79, no. 1, pp. 14-21, 2008.
[43] N. Polascheck, M. Bankstahl, and W. Loscher, "The COX-2 inhibitor parecoxib is neuroprotective but not antiepileptogenic in the pilocarpine model of temporal lobe epilepsy," Experimental Neurology, vol. 224, no. 1, pp. 219-233, 2010.

[44] K. Okada, T. Yuhi, S. Tsuji, and U. Yamashita, "Cyclooxygenase-2 expression in the hippocampus of genetically epilepsy susceptible El mice was increased after seizure," Brain Research, vol. 894, no. 2, pp. 332-335, 2001.

[45] E. Candelario-Jalil and O. Sonia Leon, "Effects of nimesulide on kainate-induced in vitro oxidative damage in rat brain homogenates," BMC Pharmacology, vol. 3, no. 1, p. 7, 2003.

[46] R. Citraro, A. Leo, R. Marra, G. De Sarro, and E. Russo, “Antiepileptogenic effects of the selective COX-2 inhibitor etoricoxib, on the development of spontaneous absence seizures in WAG/Rij rats," Brain Research Bulletin, vol. 113, pp. 1-7, 2015.

[47] M. S. Oliveira, A. F. Furian, L. M. Rambo et al., "Modulation of pentylenetetrazol-induced seizures by prostaglandin E2 receptors," Neuroscience, vol. 152, no. 4, pp. 1110-1118, 2008.

[48] A. Zandieh, F. Maleki, A. Hajimirzabeigi, B. Zandieh, O. Khalilzadeh, and A. R. Dehpour, "Anticonvulsant effect of celecoxib on pentylenetetrazole-induced convulsion: modulation by NO pathway," Acta Neurobiologiae Experimentalis, vol. 70, no. 4, pp. 390-397, 2010. 